\title{
Electronic and Conventional Cigarette Use and Drinking Behaviors in Korean Adolescents
}

\author{
Young Gyu Cho* \\ Department of Family Medicine, Inje University Seoul Paik Hospital, Inje University College of Medicine, Seoul, Korea
}

\section{See original paper on 241}

Electronic cigarettes (e-cigarettes), battery-operated devices that deliver nicotine vapor, were introduced to Korea in 2007. E-cigarette use has rapidly increased among adolescents in a number of countries. E-cigarettes have recently become the most popular tobacco product among US adolescents. ${ }^{1)}$ On the other hand, the prevalence of e-cigarette use among Korean adolescents has been stable at a low level. Since 2016, current e-cigarette users have accounted for less than $3 \%$ of Korean adolescents. ${ }^{2)}$ The different trends in e-cigarette use between US and Korean adolescents may be explained by the different regulatory environments of the two countries. Korea has implemented more extensive regulation of e-cigarettes. ${ }^{3)}$ In addition, Naver, the most representative portal site in Korea, was found to provide more negative messages on e-cigarettes than Google. Exposure to negative information on ecigarettes can increase the likelihood of having a negative view of e-cigarettes. ${ }^{4)}$

Adolescents use e-cigarettes for a variety of reasons including curiosity, good flavors, friends' use of them, being healthier than conventional cigarettes, being able to use them anywhere, the lack of a bad smell, etc. ${ }^{5)}$ But what are the main reasons that Korean adolescents use e-cigarettes? According to the 13th Korea Youth Risk Behavior Web-based Survey, 2017, the most common reason for adolescents to use e-cigarettes was curiosity (24.1\%). Other common reasons for using e-cigarettes were the lower harm than conventional cigarettes (18.8\%), good flavors (12.0\%), improved taste (11.2\%), being able to use them indoors (8.4\%), helping to quit conventional cigarette smoking (8.1\%), and the lack of a bad smell (8.1\%). Lee et al. ${ }^{7}$ performed in-depth interviews with 11 Korean male high school students who had e-cigarette experience. The students said that the biggest merit of e-cigarettes was being able to use them anywhere because of the lack of odor. Even though they perceived e-cigarettes as less harmful than conventional cigarettes, few students used e-cigarettes for health benefits. Some decided to use e-cigarettes to save money by quitting smoking, as the price of conventional cigarettes has increased. However, they were not fully satisfied with e-cigarettes and became dual users. They usually used ecigarettes at school or home and smoked conventional cigarettes outdoors.

E-cigarette companies have advertised e-cigarettes as safer alternatives to conventional cigarettes and effective smoking cessation aids. ${ }^{8)}$ Responding to this marketing, some Korean adolescents may use e-cigarettes to quit smoking. However, it was shown that successful smoking cessation through e-cigarettes was rare among adolescents. ${ }^{9)}$ Kalkhoran and Glantz ${ }^{10)}$ reported that e-cigarette use was associated with a $28 \%$ lower likelihood of quitting smoking in the real world even among adults. Whereas e-cigarette companies have claimed that they sell e-cigarettes as effective tools for smoking cessation, their devices are mainly consumed for recreational purposes in the real world. Many health professionals are concerned that ecigarettes could act as a gateway to smoking among young non-smokers. E-cigarettes, perceived as harmless and attractive, are more likely to be tried by adolescents who do not dare to smoke conventional cigarettes. Once addicted to nicotine, they could move on to conventional cigarettes easily. ${ }^{11)}$ Early 
exposure to nicotine through e-cigarette use during adolescence may induce epigenetic changes that sensitize the brain to other substances. So, e-cigarettes are a possible gateway to the use of not only conventional cigarettes but also other substances. ${ }^{12)}$

Regarding the present issue, Oh et al. ${ }^{13)}$ investigated whether e-cigarette use is related to drinking behaviors regardless of conventional cigarette use in Korean adolescents using the data from the 11th Korea Youth Risk Behavior Web-based Survey, 2015. The study participants consisted of 68,043 students (35,204 males and 32,839 females) from middle and high schools in Korea. In their study, e-cigarette-only users were more likely to be current drinkers (odds ratio [OR], 6.5 in males and 10.8 in females) and problem drinkers (OR, 7.4 in males and 14.5 in females) compared to students who had tried neither product. Dual users, who used both e-cigarettes and conventional cigarettes, had a higher likelihood of current drinking (OR, 2.1 in males and 1.5 in females) and problem drinking (OR, 1.9 in males and 1.3 in females) than conventional cigarette-only users. These results imply that e-cigarettes and conventional cigarettes have additive negative effects on risky drinking behaviors.

The finding that e-cigarette use is closely linked to risky drinking behaviors is consistent with the results of previous studies. Hughes et al. ${ }^{14)}$ explored the association between e-cigarette access and drinking behaviors among adolescents in England. In the study, binge drinkers had higher odds of accessing e-cigarettes compared to non-drinkers. Among drinkers, e-cigarette access was associated with the self-purchase of alcohol from off-licensed premises and the recruitment of proxy purchasers from outside premises. This result means that adolescents who access e-cigarettes are likely to already be familiar with strategies to bypass regulations on age-restricted products. E-cigarette use in adolescence was reported to be related to not only binge drinking but also the use of marijuana, amphetamines, and other illicit substances, plus the misuse of prescription medications. ${ }^{15,16)}$ Multiple adolescent problem behaviors including smoking, drinking, and substance use are likely to co-occur as a result of shared common predisposing factors. As e-cigarette use is related to an increased risk of multiple adolescent problem behaviors, e-cigarette use should be regarded as a serious problem behavior common to adolescents.

Many adolescents still believe that e-cigarettes are harmless and can be used as effective smoking cessation aids. However, e-cigarettes are not safe and are not helpful when quitting smoking, especially among adolescents. ${ }^{17)}$ In fact, e-cigarettes can serve as a gateway to the use of conventional cigarettes, alcohol, and other substances. In order to protect adolescents from harm caused by e-cigarettes, we should develop and implement educational campaigns to correct their wrong beliefs about e-cigarettes and portray them as a potential gateway to more harmful substances.

\section{CONFLICT OF INTEREST}

No potential conflict of interest relevant to this article was reported.

\section{ORCID}

Young Gyu Cho: https://orcid.org/0000-0003-1017-8884

\section{REFERENCES}

1. Singh T, Arrazola RA, Corey CG, Husten CG, Neff LJ, Homa DM, et al. Tobacco use among middle and high school students: United States, 2011-2015. MMWR Morb Mortal Wkly Rep 2016;65:361-7.

2. Ministry of Education, Ministry of Health and Welfare, Korea Centers for Disease Control and Prevention. The 14th Korea Youth Risk Behavior Web-based Survey, 2018. Cheongju: Korea Centers for Disease Control and Prevention; 2018.

3. Cho HJ, Dutra LM, Glantz SA. Differences in adolescent e-cigarette and cigarette prevalence in two policy environments: South Korea and the United States. Nicotine Tob Res 2018;20:949-53.

4. Kim SJ, Park EW, Choi EY, Cheong YS. Comparison of information between domestic and international web-pages for e-cigarettes: what are the differences between Naver and Google? Korean J Health Promot 2019;19:25-31.

5. Bold KW, Kong G, Cavallo DA, Camenga DR, Krishnan-Sarin S. Reasons for trying e-cigarettes and risk of continued use. Pediatrics 2016;138:e20160895.

6. Ministry of Education, Ministry of Health and Welfare, Korea Centers for Disease Control and Prevention. The 13th Korea Youth Risk Behavior Web-based Survey, 2017. Cheongju: Korea Centers for Disease Control and Prevention; 2017.

7. Lee YR, Kimm H, Lee S, Jeon J, Chu S, Jee SH. Associations between the attempts for quitting smoking and electronic cigarette use in Korean adolescent. Korean J Health Educ Promot 2017;34:71-83.

8. Lee S, Kimm H, Yun JE, Jee SH. Public health challenges of electronic cigarettes in South Korea. J Prev Med Public Health 2011;44:235-41.

9. Lee S, Grana RA, Glantz SA. Electronic cigarette use among Korean adolescents: a cross-sectional study of market penetration, dual use, and relationship to quit attempts and former smoking. J Adolesc Health 2014;54:684-90.

10. Kalkhoran S, Glantz SA. E-cigarettes and smoking cessation in realworld and clinical settings: a systematic review and meta-analysis. Lancet Respir Med 2016;4:116-28.

11. Akre C, Suris JC. Adolescents and young adults' perceptions of electronic cigarettes as a gateway to smoking: a qualitative study in Switzerland. Health Educ Res 2017;32:448-54.

12. Yuan M, Cross SJ, Loughlin SE, Leslie FM. Nicotine and the adolescent brain. J Physiol 2015;593:3397-412.

13. Oh KH, Lee CM, Oh B, Oh SW, Joh HK, Choi HC, et al. The relationship between electronic cigarette use with or without cigarette smoking and alcohol use among adolescents: finding from the 11th Korea Youth Risk Behavior Web-based Survey. Korean J Fam Med 2019;40: 241-7.

14. Hughes K, Bellis MA, Hardcastle KA, McHale P, Bennett A, Ireland R, Pike K. Associations between e-cigarette access and smoking and drinking behaviours in teenagers. BMC Public Health 2015;15:244.

15. Kristjansson AL, Mann MJ, Sigfusdottir ID. Licit and illicit substance use by adolescent e-cigarette users compared with conventional cigarette 
smokers, dual users, and nonusers. J Adolesc Health 2015;57:562-4.

16. McCabe SE, West BT, Veliz P, Boyd CJ. E-cigarette use, cigarette smoking, dual use, and problem behaviors among U.S. adolescents: results from a national survey. J Adolesc Health 2017;61:155-62.

17. Glantz SA, Bareham DW. E-cigarettes: use, effects on smoking, risks, and policy implications. Annu Rev Public Health 2018;39:215-35. 